\title{
Neurological manifestations of HIV-AIDS at a tertiary care center in western Maharashtra
}

\section{Virendra C. Patil, Harsha V. Patil ${ }^{1}$}

Departments of Medicine and ${ }^{1}$ Microbiology, Krishna Institute of Medical Sciences University (KIMSU), Satara, Maharashtra, India

Address for the Correspondence: Dr. Virendra C. Patil, Department of Medicine, Krishna Institute of Medical Sciences University (KIMSU), Karad,

Dhebewadi Road,

Satara - 415110

Maharashtra, India.

E-mail: virendracpkimsu@ rediffmail.com

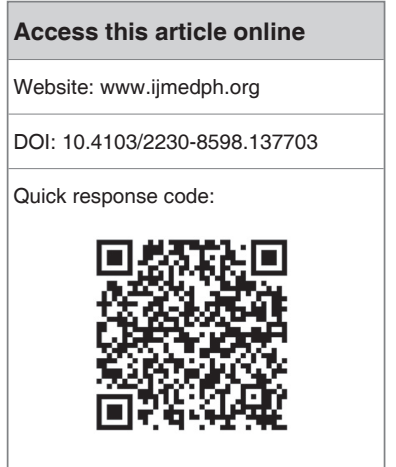

Background: There is an increasing incidence of patients infected with human immunodeficiency virus (HIV) in India. The neurological manifestations of the disease are being seen more frequently. The nervous system is the most frequent and serious target of HIV infection. Aims and Objectives: To elucidate the spectrum of neurological involvement in patients with HIV infection at a tertiary care teaching hospital in western Maharashtra. We investigated various neurological manifestations of HIV including opportunistic infections (OPIs) and non-opportunistic infections (nonOPIs). Settings and Design: This was a retrospective observational study conducted at a tertiary care center in western Maharashtra over a period of 2 years from Jan 2009 to Dec 2010. Materials and Methods: A total of 81 HIV seropositive patients of both genders, of age $>18$ years, with neurological manifestations admitted at a tertiary care center were studied for clinical parameters, laboratory investigations and imaging. Statistical Analysis: Data were coded by numbers and double entered in a computer software SSPE-11 trial version. Results: A total of 179 patients admitted with HIV infection, of which 81 (45.25\%) presented with neurological manifestations (neuro-acquired immunodeficiency syndrome [AIDS]), were enrolled in the study. Overall, 53 (65.43\%) patients were male (34 years \pm 11 ) and 28 (34.56\%) were female (29 years \pm 8 ). The male patients were outnumbered compared with the female patients, with $P=0.02$. A total of $45(55.55 \%)$ patients had OPIs and 36 $(44.44 \%)$ patients had non-oppurtunistic neurological manifestations affecting the nervous system ( $P=1573$; insignificant). A total of $15(18.51 \%)$ patients had immune reconstitution syndrome on antiretroviral therapy (A total of $11(13.58 \%$ ) patients had seizures, eight $(9.87 \%)$ had ischemic stroke, eight $(9.87 \%)$ had aseptic meningitis, two $(2.46 \%)$ had intracranial hemorrhage, two $(2.46 \%)$ had vacuolar myelopathy, four $(4.93 \%)$ had AIDS-associated dementia, three $(3.70 \%)$ had Guillain Barré syndrome (GBS), two (2.46\%) had acute motor sensory axonal neuropathy (AMSAN), one $(1.23 \%)$ had chronic inflammatory demyelinating polyradiculoneuropathy (CIDP) and two $(2.46 \%)$ had mononeuritis multiplex cranialis. A total of 17 (20.98\%) patients had TB meningitis, $11(13.58 \%)$ had cryptoccocal meningitis, one $(1.23 \%)$ had Pott's spine, two $(2.46 \%)$ had progressive multifocal leukoencephalopathy (PMLE), two $(2.46 \%)$ had herpes zoster, one $(1.23 \%)$ had herpes simples encephalitis and one $(1.23 \%)$ had cerebral toxoplasmosis. The CD4 was significantly low in patients with PMLE, ADC (AIDS Dementia Complex) and cryptoccocal meningitis compared with other neurological manifestations $(P<$ $0.002)$. The case fatality rate was $7.4 \%(6 / 81)$. Mortality was significantly high in patients with cryptoccocal meningitis and PMLE compared with the other neurological manifestations ( $P=0.034)$. Conclusion: We found a high prevalence of neurological manifestations in HIV seropositive patients $(45.25 \%)$ in this setting. Central nervous system (CNS) tuberculosis was the most common secondary infection seen in HIV patients. Cryptococcal meningitis was the next common infection, which showed a striking male preponderance. The most common non-infectious lesions included cerebrovascular events, followed by neoplasms. Neuropathies and myelopathies were the least common neurological manifestations in patients with HIV infection. This study revealed not only the high prevalence of various neurologic events but also their nature, clinical presentation and symptoms. A neuropsychological assessment should be mandatory for all HIV-positive patients. CNS OPI indicates progression of HIV infection toward AIDS, and is useful as a reference to starting ART in settings where facilities for determination of CD4 counts are not available.

Key words: Cryptoccocal meningitis, immune reconstitution syndrome, non-opportunistic neurological manifestations, opportunistic infections, TB meningitis 


\section{INTRODUCTION}

The incidence of human immunodeficiency virus (HIV) is increasing in India, and central nervous system (CNS) manifestations of the disease are being seen more frequently. ${ }^{[1]}$ The later stages of HIV cause severe immunodeficiency and render the patient susceptible to an array of neurological disorders, affecting virtually every component of the nervous system, and lead to considerable morbidity and mortality. At least $40 \%$ of HIV-infected patients develop neurological symptoms during the course of their illness. HIV infection is responsible for a large number of nonopportunistic neurologic manifestations that occur across a large immune spectrum. During the early course of the disease, the polyclonal hypergammaglobulinemia induced by the virus results in demyelinating diseases of the CNS and peripheral nervous system (PNS). As the HIV infection progresses, the direct toxic effects of the virus unfold, directly damaging the CNS and PNS, resulting in protean clinical manifestations. ${ }^{[1]}$ Neurological disease is the presenting manifestation of acquired immunodeficiency syndrome (AIDS) in $10-20 \%$ of patients. Autopsy studies have revealed neuropathological abnormalities in $80-90 \%$ of patients dying with AIDS. Thus, the knowledge of CNS manifestations of HIV is very important to the clinician. With the continued widespread use of combination antiretroviral therapy (ART), the incidence of various neurological complications remains low. However, some complications continue to have a serious impact on the lives of HIV-infected patients. HIV-positive people continue to live longer because of ART, as the risk of neurological complications stemming from comorbidities increases. The Indian population is primarily infected with subtype "C" HIV-1..$^{[1,2]}$ Neurologic manifestations affecting the nervous system at all levels and stages of HIV infection are common and increasing with the extended survival of HIV-positive persons. We know that in the last few years, HIV/AIDS has become a chronic manageable disease instead of an incurable disease. Thus far, there are limited studies available about the neurological manifestations in neuro-AIDS in India. This retrospective observational study was conducted to document the neurologic events in HIV cases in western Maharashtra.

\section{MATERIALS AND METHODS}

This was a retrospective observational study conducted at a tertiary care center in western Maharashtra over a period of 2 years from Jan 2009 to Dec 2010. The protocol was approved by the Ethical committee of the Krishna Institute of Medical Sciences, Karad. Of a total of 179 patients with HIV-AIDS, 81 seropositive patients of both genders aged $>18$ years with neurological manifestations (neuro-AIDS) admitted at a tertiary care center were included in this study. The mean duration of neurological manifestation to diagnosis of HIV infection was 6-18 months in non-opportunistic infections (non-OPIs) and 3-5 years for opportunistic infections (OPIs) in the current study. Case records of HIV-positive patients with CNS involvement admitted in the Krishna Institute of Medical Sciences, Karad, were studied with respect to demographic data (age, sex, etc.), history, clinical examination, laboratory tests including complete blood count, liver and kidney function tests, cerebrospinal fluid (CSF) examination (proteins, sugar, chlorides, cytology), chest $\mathrm{X}$-ray, special staining studies (Ziehl - Neelsen stain, India ink, etc.), specific antigen detection tests in serum and CSF (cryptococcal and toxoplasmosis antigen) and computed tomography (CT) brain and magnetic resonance imaging (MRI) brain/spine scan. Specific opportunistic infections were diagnosed on the basis of standard clinical definitions and laboratory procedures. Viral load estimation was not performed because of financial constraints.

Diagnosis of HIV was confirmed by enzyme-linked immunosorbent assay (ELISA) using two different antigens and a rapid test as recommended by the National AIDS Control Organization (NACO). After confirmation of HIV infection by the VCTC center in the Department of Microbiology of the institute, CD4 count was done.

\section{AIMS AND OBJECTIVES}

To study the clinical and laboratory profiles of HIV-infected patients with neurological manifestations in a tertiary care teaching hospital.

All HIV-infected patients confirmed by HIV ELISA who presented with neurological manifestations at a tertiary care center in western Maharashtra, India, were subjected to thorough neurological evaluation. Wherever indicated, neuro-imaging, cerebrospinal fluid study, electromyography and nerve conduction studies were performed to confirm the diagnosis. CD4 count was measured using standard flow cytometry. All the enrolled participants were screened for differential detection of HIV 1 and HIV 2 antibodies using a highly sensitive, visual and rapid immunoassay (HIV TRIDOT). Patients testing positive in the initial screening test for either HIV 1 or HIV 2 were subjected to two different confirmatory ELISA tests using two different types of antigens, as recommended by the NACO. Opportunistic infections like tuberculosis were diagnosed by using a combination of imaging and Ziehl — Neelsen staining. Cryptococcal meningitis was ruled out using CSF for India ink and fungal culture. Other relevant investigations including CT head, MRI imaging, CSF examination, complete blood counts, kidney function and liver function tests were performed as and when dictated by clinical presentation of the patient. All patient information was kept highly confidential. ${ }^{[3-5]}$ All the patients included in the study had absolute CD4+ levels documented whenever possible. For clinical staging of these patients, we used the " 1993 revised classification system for HIV infection and expanded surveillance case definition for AIDS among adolescents and adults." Patients with a diagnosis of neuro-AIDS were treated according to the NACO guidelines in the form of ART, anti-tubercular drugs, antifungal, decongestive, antibiotics and anticonvulsants, and associated OPIs were treated with respective drugs and mechanical ventilation etc. were used whenever required. Symptomatic and asymptomatic HIV-infected patients with a CD4 count $<350 / \mathrm{Pl}$ were put on highly active antiretroviral therapy (HAART) as recommended..$^{[3-5]}$ 
Chemoprophylaxis and ART was advised as indicated. Patients were offered ART if their CD4 lymphocyte count was less than $<350$ cells/mL. Trimethoprim — sulfamethoxazole prophylaxis was given to prevent Pneumocystis jiroveci pneumonia in all patients with a CD4 lymphocyte count of $<200$ cells/mL.

IRIS: ART initiation in HIV-infected patients leads to recovery of $\mathrm{CD} 4+\mathrm{T}$ cell numbers and restoration of protective immune responses against a wide variety of pathogens, resulting in reduction in the frequency of OPIs and prolonged survival. However, in a subset of patients, dysregulated immune response after initiation of ART leads to the phenomenon of immune reconstitution inflammatory syndrome (IRIS). The hallmark of the syndrome is paradoxical worsening of an existing infection or disease process or appearance of a new infection/disease process soon after initiation of therapy.

\section{Statistical analysis}

Data were coded and double entered in a computer software SSPE11 trial version. Results were given as mean \pm SD. Means were compared using unpaired Students $t$-test. Chi-square was used as appropriate. The level of significance was set at $P<0.05$.

\section{RESULTS}

A total of 179 patients were admitted with HIV infection, of whom $81(45.25 \%)$ presented with neurological manifestations. A total of $53(65.43 \%)$ were male $(34$ years \pm 11$)$ and $28(34.56 \%)$ were female (29 years \pm 8 ). The male patients were outnumbered compared with the female patients. with $P=0.02$. Overall, $45(55.55 \%)$ patients had opportunistic (OPI) neurological manifestations and $36(44.44 \%)$ patients had non-OPI affecting the nervous system $(P=0.1573$; insignificant). A total of $15(18.51 \%)$ patients had immune reconstitution syndrome on ART. Male patients were affected more often than female patients in neurological manifestation in HIV-AIDS, with $P<0.02$. The mean duration of presentation of neurological manifestation from the diagnosis of HIV infection was 36 months $( \pm 17)$. The mean duration of neurological manifestation to diagnosis of HIV infection was 18-24 months in non-OPIs and 36-48 months for OPIs in the current study [Table 1].

\section{Neuro-AIDS with non-OPIs}

Conditions included in non-OPIs were seizures, ischemic stroke, aseptic meningitis, intracranial hemorrhage, vacuolar myelopathy, AIDS-associated dementia, GB syndrome, AMSAN, CIDP, mononeuritis multiplex cranialis and dilated cardiomyopathy. A total of $11(13.58 \%)$ patients had seizures, eight $(9.87 \%)$ had ischemic stroke, eight $(9.87 \%)$ had aseptic meningitis, two $(2.46 \%)$ had intracranial hemorrhage, two $(2.46 \%)$ had vacuolar myelopathy, four $(4.93 \%)$ had AIDS-associated dementia, three (3.70\%) had GB syndrome, two $(2.46 \%)$ had AMSAN, one $(1.23 \%)$ had CIDP and two $(2.46 \%)$ had mononeuritis multiplex cranialis. Ischemic stroke was the most common neurological manifestation among the non-OPIs, with $P<0.05$. A 45-year-old male patient in our study presented with breathlessness (NYHA class-III) and developed embolic stroke after an episode of ill-sustained ventricular tachycardia had echocardiographic features suggestive of dilated cardiomyopathy with echo-contrast in the left ventricle [Tables 2 and 4] [Figures 2 and 4] [Graph 1].

\section{Neuro-AIDS with OPI}

Conditions included in OPIs were TB meningitis, cryptoccocal meningitis, Pott's spine, multifocal leukoencephalopathy (PMLE), herpes zoster, herpes simplex and cerebral toxoplasmosis. A total of $17(20.98 \%)$ patients had TB meningitis, 11 (13.58\%) had cryptoccocal meningitis, one (1.23\%) had Pott's spine (D-9-10 Pott's spine with compressive myelopathy causing subacute sensory motar paraplegia with bladder involvement with pulmonary tuberculosis),

\begin{tabular}{|c|c|c|}
\hline Variables & Total number & Percent \\
\hline Male & 53 & 65.43 \\
\hline Female & 28 & 34.56 \\
\hline OPIs & 45 & 55.55 \\
\hline Non-OPIs & 36 & 44.44 \\
\hline IRIS & 15 & 18.51 \\
\hline
\end{tabular}

\begin{tabular}{|c|c|c|c|}
\hline Variables & Total $(n=27)$ & Percent & CD4/microL $(n=14)$ \\
\hline Seizure & 11 & 13.58 & $n=7(213 \pm 23)$ \\
\hline Ischemic stroke & 8 & 9.87 & $\mathrm{n}=4(195 \pm 12)$ \\
\hline IC bleed & 2 & 2.46 & $\mathrm{n}=1$ (198) \\
\hline $\mathrm{HAD}$ & 4 & 4.93 & $n=2(95 \pm 15)$ \\
\hline Vacuolar myelopathy & 2 & 2.46 & - \\
\hline
\end{tabular}

\begin{tabular}{|c|c|c|c|}
\hline Variables & Total $(n=45)$ & Percent & CD4/microL $(n=19)$ \\
\hline Aseptic meningitis & 10 & 12.34 & $\mathrm{n}=5(210 \pm 32)$ \\
\hline TBM & 17 & 20.98 & $\mathrm{n}=7(55 \pm 12)$ \\
\hline Cryptococcal & 12 & 14.81 & $\mathrm{n}=6(42 \pm 9)$ \\
\hline HSV & 1 & 1.234 & - \\
\hline $\mathrm{HZV}$ & 2 & 2.469 & - \\
\hline Toxoplasmosis & 1 & 1.234 & - \\
\hline Pott's spine & 1 & 1.234 & - \\
\hline PMLE & 1 & 1.234 & 23 \\
\hline
\end{tabular}

Table 4: Distribution of central and peripheral nervous system in non-OPIs of HIV-AIDS patients

\begin{tabular}{lccc}
\hline Variables & Total $(\mathbf{n}=\mathbf{9})$ & Percent & CD4/microL $(\mathbf{n}=\mathbf{2})$ \\
\hline GBS & 3 & 3.70 & $\mathrm{n}=2(188 \pm 20)$ \\
AMSAN & 2 & 2.46 & - \\
CIDP & 2 & 2.46 & - \\
Mononeuritis multiplex & 2 & 2.46 & - \\
cranialis & & & \\
\hline
\end{tabular}


one $(1.23 \%)$ had PMLE, two $(2.46 \%)$ had herpes zoster (one with left ophthalmic division of trigeminal nerve and the other with T3-T4 thoracic dermatomes on the left side presented with thoracic radicular pain), one (1.23\%) had herpes simplex (bilateral temporal lobe involvement in herpes simplex presented with altered sensorium and seizures) encephalitis and one (1.23\%) had cerebral toxoplasmosis (presented with left focal seizures with secondary generalization). Tubercular meningitis was the most common OPI in neurological manifestation, with $P<0.02$. PMLE was the least common neurological manifestation among OPIs. The patient with an MRI finding suggestive of PMLE was a 45 -year-old male on ART for a 6-month duration, who had a CD4+ count 23 presented with progressive deterioration of cognitive and motor function over 1 month and succumbed with 5 days of admission [Tables 3 and 4] [ Figures 1-3 and 5] [Graph 1].

\section{CD4+ counts}

A total of $35(43.20 \%)$ patients with neurological manifestations underwent CD4 count. Overall, 19 (42.22\%) patients with OPIs and $16(44.44 \%)$ patients with non-OPIs underwent CD4 count. The mean CD4 count was lowest in patients with PMLE among neurological manifestations of HIV-AIDS in OPIs, followed by cryptococcal meningitis. The CD4 count was significantly low in patients with PMLE, ADC and cryptoccocal meningitis compared

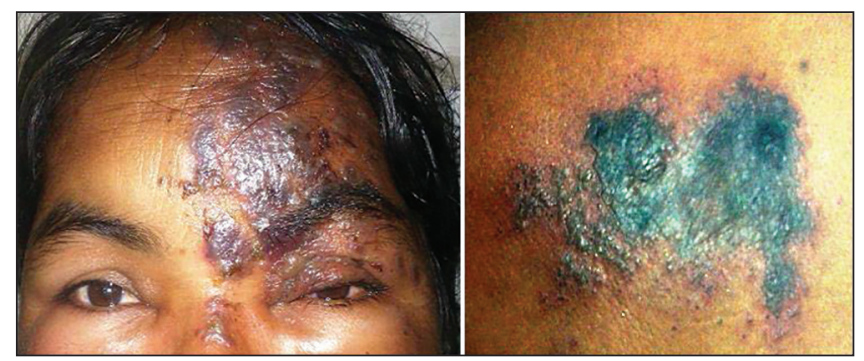

Figure 1: Herpes zoster involving the left ophthalmic division of the trigeminal nerve and T3-T4 thoracic dermatomes on the left side

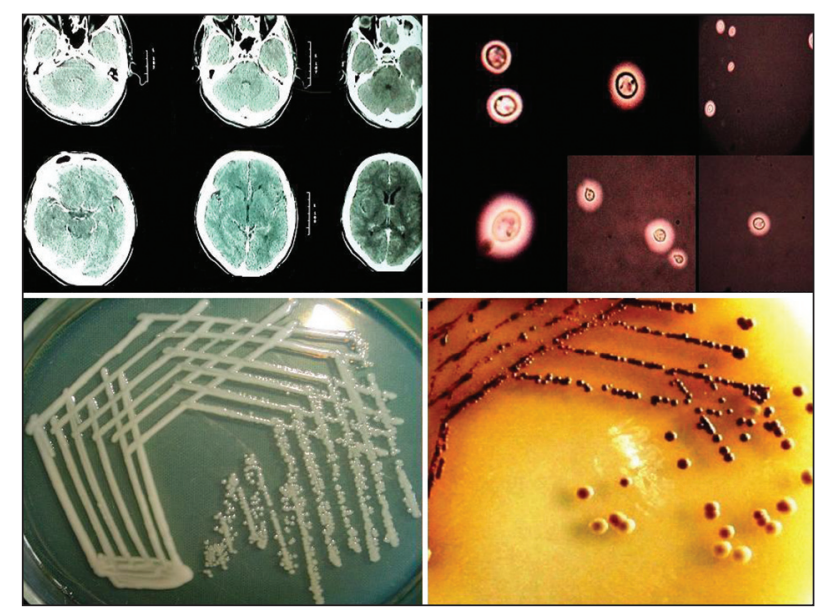

Figure 3: Computed tomography of the brain showing cerebral edema with compressed ventricles in a patient with cryptococcal meningitis with nigrosin staining showing Cryptococcus neoformans and cerebrospinal fluid culture colonies on Sabouraud dextrose agar and Niger seed agar with the other neurological manifestations $(P<0.002)$. The mean CD 4 count was lowest in patients with HAD among neurological manifestation of HIV-AIDS in non-OPIs. CD4 counts were lowest in the patient with PMLE (23/microL) and cryptococcal meningitis $(42 \pm 9)$. The mean CD4 count was lowest in the patient with HAD among neurological manifestations of HIV-AIDS in non-OPIs.

\section{Patients on ART}

A total of 50.61\% (41) patients were on ART — two with CIDP, two with GB syndrome, two with AMSAN, five with aseptic meningitis, nine with tubercular meningitis (TBM), seven with cryptoccocal meiningitis, one with herpes simplex encephalitis, one with PMLE, six with seizures, three with ischemic stroke, one with IC bleed and two with HIV-associated dementia were on ART. A total of 23 nonOPIs and 18 OPIs were on ART. Statistically, there was no relation between the ART status and the development of neurological manifestations in HIV/AIDS patients. There is no temporal relation of neurological manifestations and patients on ART.

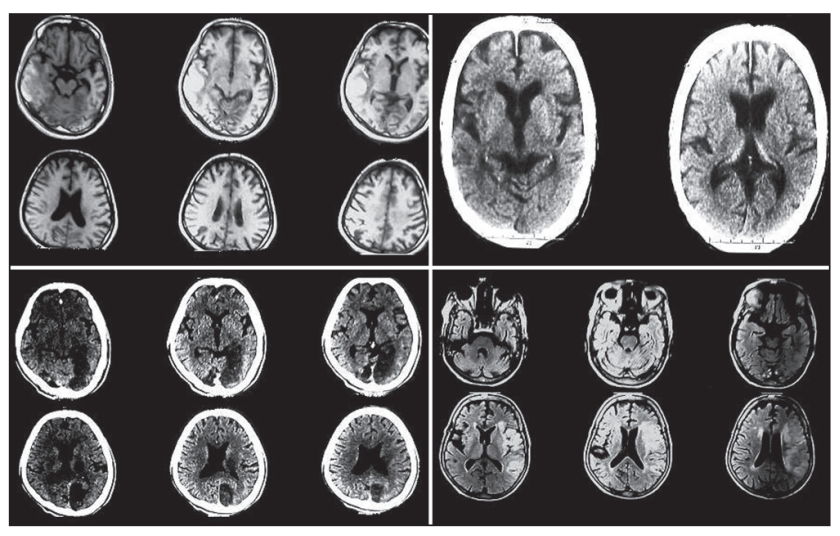

Figure 2: Magnetic resonance imaging (MRI) showing bilateral temporal lobe involvement in herpes simplex; computed tomography (CT) brain showing the acquired immunodeficiency syndrome demetia complex; CT brain showing left middle cerebral and posterior cerebral artery territery infarct; MRI showing progressive multifocal leukoencephalopathy

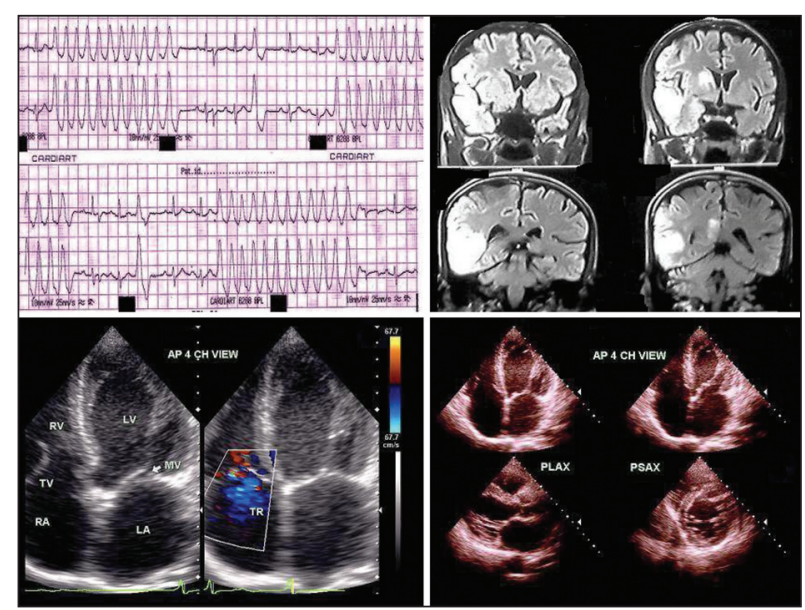

Figure 4: Electrocardiogram showing ill-sustained ventricular tachycardia with echocardiogram showing features of dilated cardiomyopathy with embolic stroke causing right MCA territery infarct 


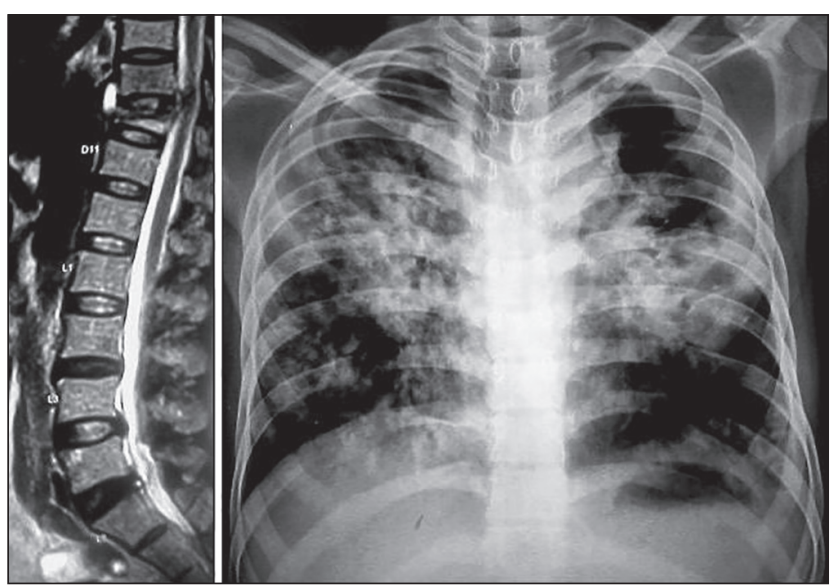

Figure 5: Magnetic resonance imaging of the spine showing thoracic D-9-10 Pott's spine with pulmonary tuberculosis

IRIS in patients with neuro-AIDS: A total of $36.58 \%$ (15) patients on ART developed IRIS. IRIS was more prevalent in patients with seizures on ART, with $P<0.05$. There is a temporal relation of neurological manifestations and patients on ART developing IRIS [Table 6].

\section{Mortality in patients with neuro-AIDS}

All death in patients with a neurological manifestation of HIV-AIDS had OPIs. The overall mortality rate in patients with neuro-AIDS was $7.4 \%$ (6/81). Mortality was significantly high in patients with cryptoccocal meningitis and PMLE compared with other neurological manifestations $(P=0.034)$. The case fatality rate in PMLE was $100 \%$, in cryptoccocal meningitis was $25 \%$ and in TBM was $11.76 \%$. The case fatality rate was highest in PMLE (100\%) [Table 5].

\section{DISCUSSION}

Neurologic manifestations affecting the nervous system at all levels and stages of HIV infection are common and increasing with the extended survival of HIV-positive persons in the last decade. The combinatorial ART has widely declined the number of HIV-related deaths, and resulted in an increase in number of people living with HIV and their morbidity in terms of their compromised brain functions, thereby worsening the overall scenario in the form of HIV-associated neurocognitive impairment that are studied under the umbrella of neuro-AIDS. Productively infected macrophages "hijack" brain parenchyma, resulting in slow neurodegeneration, especially in the basal ganglia, hippocampus, pre-frontal cortex and white matter. In addition, more devastating is the condition when HIV synergizes with drugs of abuse and brings in oxidative stress, elevation of inflammatory cytokines and increased calcium waves, ultimately leading to augmented excitotoxicity. ${ }^{[6]}$

We compared our results with various other studies in the Indian and western literature. Kumarasamy et al. ${ }^{[7]}$ stated that the mean duration of survival after diagnosis with HIV in India is 92 months. With CD4 counts less than 200 cells/ $\mu$ l, patients are at high risk for developing

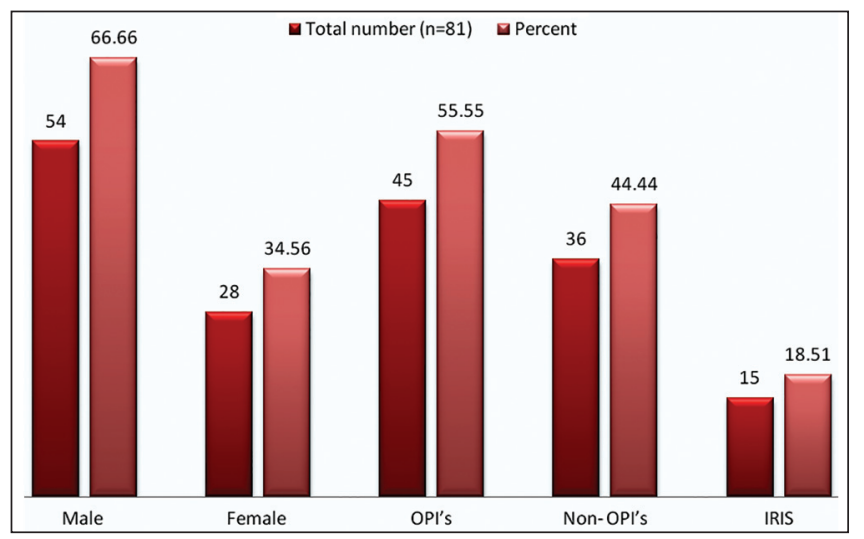

Graph 1: Prevalence of opportunistic infections and non-opportunistic infections in patients with neurological manifestations in neuro-acquired immunodeficiency syndrome

\begin{tabular}{lcc}
$\begin{array}{l}\text { Table 5: Mortality in a patient with neurological } \\
\text { manifestation of HIV-AIDS }\end{array}$ & Total $(\mathbf{n}=\mathbf{3 0})$ & Percent \\
\hline Variable & 2 & 11.76 \\
\hline TBM $(n=17)$ & 3 & 25 \\
Cryptococcal meningitis $(n=12)$ & 1 & 100 \\
PMLE $(n=1)$ & 6 & 20 \\
Total & \\
\hline
\end{tabular}

\begin{tabular}{|c|c|c|c|c|}
\hline Variables & ART & Percent & IRIS & Percent \\
\hline GBS $(n=3)$ & 2 & 66.66 & - & - \\
\hline AMSAN $(n=2)$ & 2 & 100 & - & - \\
\hline $\operatorname{CIDP}(n=2)$ & 2 & 100 & 1 & 50 \\
\hline $\begin{array}{l}\text { Mononeuritis multiplex } \\
\text { cranialis }(n=2)\end{array}$ & - & - & & - \\
\hline Aseptic meningitis $(n=10)$ & 5 & 50 & 2 & 40 \\
\hline TBM $(n=17)$ & 9 & 52.94 & 3 & 33.33 \\
\hline Cryptococcal $(n=12)$ & 7 & 58.33 & 2 & 28.57 \\
\hline $\operatorname{HSV}(n=2)$ & 1 & 50 & 1 & - \\
\hline $\mathrm{HZV}(\mathrm{n}=1)$ & - & - & - & - \\
\hline Toxoplasmosis $(n=1)$ & - & - & - & - \\
\hline Pott's spine $(n=1)$ & - & - & - & - \\
\hline PMLE $(n=1)$ & 1 & 100 & - & - \\
\hline Seizure $(n=11)$ & 6 & 54.54 & 4 & 66.66 \\
\hline Ischemic stroke $(n=8)$ & 3 & 37.5 & 1 & 33.33 \\
\hline IC bleed $(n=2)$ & 1 & 50 & - & - \\
\hline $\operatorname{HAD}(n=4)$ & 2 & 50 & 1 & 50 \\
\hline Vacuolar myelopathy $(n=2)$ & - & - & - & \\
\hline Total & 41 & 50.61 & 15 & 38.58 \\
\hline
\end{tabular}

OPIs. Similarly, in our study, in patients with OPIs with neurological manifestation in the form of TB meningitis ( $\mathrm{n}=7[55 \pm 12$ cells $/ \mu \mathrm{l}])$ and cryptococcal meningitis ( $\mathrm{n}=6[42 \pm 9$ cells/ $\mu \mathrm{l}]$ ) had low CD4 count compared with neurological manifestation in non-OPIs like seizure $(n=7[213 \pm 23])$, ischemic stroke $(n=4[195 \pm 12])$ and IC bleed ( $\mathrm{n}=1$ [198]), with $P<0.02 .{ }^{[8]}$ Neurological complications of HIV disease can be seen in $20 \%$ of outpatients in HIV clinics and 
almost half of HIV patients being treated as inpatients. Similarly, in our study, 81 (45.25\%) patients had neuro-AIDS. Neurological complications can be categorized into opportunistic infections, malignancy, AIDS-related dementia and vasculitis/stroke.

OPIs in the CNS: Cryptococcal meningitis has been reported as the most common OPI of the CNS in Indian patients with HIV. The present study shows that TB meningitis is a common CNS OPIs, with $P<0.02$, followed by cryptoccocal meningitis. CD4 counts were lowest in the patient with PMLE (23/microL) and cryptococcal meningitis $(42 \pm 9)$. PMLE was the least common neurological manifestation among OPIs. Teja et al. ${ }^{[2]}$ reported in their retrospective study of $1606 \mathrm{HIV}$-positive patients that neurologic manifestations were found in $25.6 \%$ of the study patients. Meningitis was the most common CNS infection in patients with HIV/AIDS (39.4\%). Most of these complications were observed in the advanced stages of infection. CD 4 counts were performed in $29.9 \%$ of the cases with neurologic events, where the mean CD4 count was 89 cells/microL. Similarly, in the present study, OPIs were $55.55 \%$ with TBM, present in $20.98 \%$ patients, and cryptococcal meningitis was present in $14.81 \%$ patients. The mean CD4 count was $42 \pm 9$ cells $/ \mathrm{microL}$ in cryptococcal meningitis and $55 \pm 12$ cells/microL in TB meningitis. Deshpande et al..$^{[1]}$ in their study of 300 cases reported $67(22.3 \%)$ patients with neurologic manifestations due to the HIV infection, with the brain being most commonly involved (50.7\%). The manifestations included stroke syndromes $(29.8 \%)$, demyelinating illnesses (5.9\%), AIDS dementia complex (5.9\%) and venous sinus thrombosis $(4.4 \%)$. The other manifestations seen were peripheral neuropathies $(35.8 \%$ of cases), spinal cord pathologies $(5.9 \%$ of cases) and radiculopathies (4.4\% of cases). Similarly, in our study, $11(13.58 \%)$ patients had seizures, eight $(9.87 \%)$ patients had ischemic stroke, eight $(9.87 \%)$ patients had aseptic meningitis, two (2.46\%) patients had intracranial hemorrhage, four (4.93\%) patients had AIDS-associated dementia, three (3.70\%) and 17 (20.98\%) patients had TB meningitis, $11(13.58 \%)$ patients had cryptoccocal meningitis, one (1.23\%) patient had PMLE and one (1.23\%) patient had cerebral toxoplasmosis. A total of two $(2.46 \%)$ patients had vacuolar myelopathy, three $(3.70 \%)$ patients had GB syndrome, two $(2.46 \%)$ patients had AMSAN, one (1.23\%) patient had CIDP and two $(2.46 \%)$ patients had mononeuritis multiplex cranialis, two $(2.46 \%)$ patients had herpes zoster and one (1.23\%) patient had Pott's spine. Singh et al..$^{[9]}$ studied 416 HIV-positive patients, of whom 269 were male. A total of 312 neurological events were identified in $268(64.42 \%)$ patients having evidence of neurological involvement. HIV-associated dementia was the most common cause of morbidity (33.65\%), followed by CNS infections (21.63\%). The most common CNS infection was tuberculosis (65.56\%). CD4 counts in CNS infections and HIV-associated dementia were $64.8 / \mathrm{mL}$ and $83.52 / \mathrm{mL}$, respectively. Similarly, in the present study, TBM was present in $20.98 \%$ patients and cryptococcal meningitis was present in $14.81 \%$ patients. The mean CD4 count was $42 \pm 9$ cells/microL in cryptococcal meningitis and $55 \pm 12$ cells $/ \mathrm{microL}$ in TB meningitis. A total of 81 (45.25\%) patient had neuro-AIDS in the present study. Thorat et al. ${ }^{[10]}$ studied $102 \mathrm{HIV}$-positive patients with neurological involvement, with a male to female ratio of $4.5: 1$. The diseases with infectious etiology were CNS tuberculosis (18.5\%), cryptococcal meningitis $(16.6 \%)$, toxoplasmosis (5.8\%), PMLE $(3.9 \%)$, Cytomegalovirus encephalitis $(3.9 \%)$, cerebral abscess $(2.9 \%)$ and herpes zoster $(0.9 \%)$. The non-infectious lesions included cerebrovascular events (16.6\%), neoplasms (7.8\%), AIDS dementia complex, neuropathy and demyelination (4.9\% each), seizures and encephalopathy (2.9\% each). CNS tuberculosis is the most common secondary infection seen in HIV patients. Cryptococcal meningitis is the next common infection. The most common non-infectious lesions included cerebrovascular events, followed by neoplasms, whereas neuropathies were less common. These findings are comparable with our finding, where male patients outnumbered female patients, TB meningitis was present in $20.98 \%$ and cryptococcal meningitis was present in $14.81 \%$. One $(1.23 \%)$ patient had cerebral toxoplasmosis, one (1.23\%) patient had PMLE, two $(2.46 \%)$ patients had herpes zoster, eight $(9.87 \%)$ patients had ischemic stroke, $11(13.58 \%)$ patients had seizures, four $(4.93 \%)$ patients had AIDS-associated dementia, three $(3.70 \%)$ patients had GB syndrome, two $(2.46 \%)$ patients had AMSAN and one $(1.23 \%)$ patient had CIDP. ${ }^{[11]}$ One hundred patients (95 male and five female; mean age at presentation 31.6 +/ -9.4 years) had various neurological disorders associated with HIV infection. Eighty patients belonged to group I associated with opportunistic neuroinfections and 20 patients belonged to group II associated with non-infectious neurological disorders. Cryptococcal meningitis, either alone $(\mathrm{n}=31)$ or associated with tubercular meningitis $(n=6)$, was the most common (46.3\%) etiology. In group II (19 male and one female; mean age $32.6+/-9.4$ years), two patients had cortical dementia, three patients had acute brain stem involvement, two patients had epilepsy and one patient had features suggestive of PMLE. Six patients had peripheral nervous system involvement similar to Guillain - Barre syndrome. In our study, TB meningitis was present in $20.98 \%$ patients, one $(1.23 \%$ ) patient had PMLE, cryptococcal meningitis was present in $14.81 \%$ patients, four (4.93\%) patients had AIDS-associated dementia and three $(3.70 \%)$ patients had GB syndrome. Satishchandra et al. ${ }^{[1]]}$ reported that $33(41.25 \%)$ patients from group I and one (5\%) patient from group II died during the study. Similarly, in the present study, the mortality was significantly high in patients with cryptoccocal meningitis and PMLE compared with the other neurological manifestations $(P=0.034)$. The case fatality rate in PMLE was $100 \%$, in cryptoccocal meningitis was $25 \%$ and in TBM was $11.76 \%$. The case fatality rate was highest in PMLE (100\%). The overall mortality rate in patients with neuroAIDS was 7.4\% (6/81). Gupta et al. ${ }^{[12]}$ studied 668 HIV-infected patients, of whom $48(7.2 \%)$ patients had neurological manifestations. Twenty-six $(54.2 \%)$ patients had HIV encephalopathy. Total three $(6.3 \%)$ had epilepsy, two $(4.2 \%)$ had tubercular meningitis and two (4.2\%) had progressive multi-focal encephalopathy. Similarly, in our study, TB meningitis was present in $20.98 \%$ patients, $11(13.58 \%)$ patients had seizures and one (1.23\%) patient had PMLE. Bolokadze et al. ${ }^{[13]}$ reported in their $388 \mathrm{HIV} /$ AIDS patients $(302$ men and 86 female) that neurological complications were detected in 76 patients. Tuberculosis meningitis were the most common neurological 
disorders in $26(34 \%)$ patients, followed by CNS toxoplasmosis in $17(22 \%)$ patients, cryptococcal meningitis in $11(15 \%)$ patients and PMLE in four $(5 \%)$ patients. AIDS-related dementia was detected in 18 patients $(24 \%)$. A total of $15 \%$ patients had seizures. These findings are partially comparable with our study. Wadhwa et al. ${ }^{[14]}$ studied 17 HIV-positive adults with symptoms of chronic meningitis who were investigated for fungal meningitis because of C. neoformans, and a correlation was attempted with the CD4 counts of these patients. Cryptococcal meningitis was seen in five (29.4\%) patients, tubercular meningitis in nine $(52.9 \%)$ patients and cerebral toxoplasmosis in one patient. These findings are comparable with our study. Jowi et al..$^{[15]}$ enrolled 150 of 708 hospitalized patients with HIV-seropositive patients having neurological complications with a prevalence of $21.2 \%$. The number of males was $86(57.3 \%)$ and that of females was $64(42.7 \%)$, with a M:F ratio of 1.3:1. The mean age was 38.84 years. The five most common neurological complications were cryptococcal meningitis in $33(22 \%)$ patients, encephalitis in $28(18.7 \%)$ patients, cerebral toxoplasmosis in $19(12.7 \%)$ patients, stroke in $19(12.7 \%)$ patients and tubercular meningitis in $16(10.7 \%)$ patients. Overall, 72 patients (63\%) had CD4+ counts performed. Cryptococcal meningitis patients' CD4+ count was mean 60 , median 17 , range $1-273 / \mathrm{cmm}$ and tubercular meningitis patients' CD4+ count was mean 67 , median 62 and range $12-154 / \mathrm{cmm}$. The other rare neurological manifestations included peripheral neuropathy, HIV-associated dementia and myelopathy. One hundred and eight $(72 \%)$ patients were on ART. Fourteen (9.3\%) patients died during treatment. Similarly, in our study, peripheral neuropathy, HIV-associated dementia and myelopathy were rare neurological manifestations, with an overall mortality of $7.4 \%$ (6/81). Peripheral neuropathy is common in HIV-1 infection. Peripheral neuropathies complicate all stages of the HIV-1 disease and cause considerable morbidity and disability in HIV-1 infected individuals and AIDS patients. Acute and chronic inflammatory demyelinating polyradiculoneuropathies (AIDP and CIDP) produce global limb weakness. AIDP may occur at seroconversion, and it can therefore be the initial manifestation of HIV-1 infection. CIDP generally occurs in the mid to late stages of HIV-1 infection. Mononeuropathy multiplex (MM) in the early stages of HIV-1 infection is immune mediated. ${ }^{[16]}$ In our study, three (3.70\%) patients had GB syndrome, two $(2.46 \%)$ patients had AMSAN, one $(1.23 \%)$ patient had CIDP, two $(2.46 \%)$ patients had mononeuritis multiplex cranialis and two $(2.46 \%)$ patients had herpes zoster, with one affecting the ophthalmic division of the trigeminal nerve and the other affecting the thoracic (T-3; T4). Lanjewar et al. ${ }^{[17]}$ performed a retrospective autopsy of 85 adult brains of HIV/AIDS cases for the spectrum of neuropathological brain lesions. CNS lesions were observed in 67 cases $(79 \%)$. Opportunistic infections were present in 33 cases $(39 \%)$, which included toxoplasmosis (11 cases, $13 \%$ ), tuberculosis (10 cases, $12 \%$ ) and cryptococcosis (seven cases, $8 \%$ ). Infarcts/hemorrhages were present in 13 cases (15\%). CNS tuberculosis is frequently observed in Indian AIDS cases compared with reports from industrialized countries, where its occurrence is uncommon. These findings are comparable with the present study. Braicks et al. ${ }^{[18]}$ studied 56 patients with a mean age of $39 \pm 0.7$ years and a mean CD4 + cell count of $130 \pm 166 \mathrm{CD} 4+$ cells $/ \mu$ with neuro-AIDS. The most common manifestations of neuro-AIDS were cerebral toxoplasmosis, cryptococcosis and PMLE. In our study, TB meningitis and cryptoccocal meningitis were the most common neurological manifestations and PMLE and toxoplasmosis were the least common manifestations. Braicks et al. reported a 50\% mortality in neuro-AIDS patients. The overall mortality rate in our study with neuro-AIDS was 7.4\% (6/81). ${ }^{[19]} \mathrm{HIV}$-associated polyneuropathy has become the most common neurological complication of HIV infection and is one of the main risk factors for development of a neuropathy worldwide. In our study, three $(3.70 \%)$ patients had GB syndrome, two $(2.46 \%)$ patients had AMSAN, one $(1.23 \%)$ patient had CIDP and two $(2.46 \%)$ patients had mononeuritis multiplex cranialis and two $(2.46 \%)$ patients had herpes zoster, with one affecting the ophthalmic division of the trigeminal nerve and the other affecting the thoracic (T-3; T4). Yunis et al. ${ }^{[20]}$ stated that pericardial effusion, myocarditis, cardiomyopathy, endocarditis and coronary vasculopathy are among the most commonly reported abnormalities. Sani et al. ${ }^{[1]}$ stated that heart muscle disease is the most important cardiovascular manifestation of HIV infection, and is likely to become even more prevalent as HIV-infected patients live longer. This may present as myocarditis, non-specific or infectious myocarditis, dilated cardiomyopathy with global left ventricular dysfunction, endocardial valvular disease due to marantic or infective endocarditis, arrhythmias and pulmonary hypertension. Similarly, one 45-year-old male patient in our study who presented with breathlessness (NYHA class-III) developed embolic stroke after an episode of ill-sustained ventricular tachycardia and had echocardiographic features suggestive of dilated cardiomyopathy with echo-contrast in the left ventricle.

\section{CONCLUSIONS}

We observed that a significant proportion of patients with HIVAIDS had neurological manifestations (45.25\%) in this setting. CNS tuberculosis was the most common secondary infection (OPIs) seen in HIV-AIDS patients. Cryptococcal meningitis was the next common infection. The most common non-infectious lesions included cerebrovascular events. Neuropathies and myelopathies were the least common neurological manifestations in patients with HIV infection. This study revealed not only the high prevalence of various neurologic events but also their nature, clinical presentation and symptoms. A neuropsychological assessment should be mandatory for all HIVpositive patients. CNS OPIs indicate progression of HIV infection toward AIDS and are useful as a reference to starting ART in settings where facilities for determination of CD4+ counts are not available. It is necessary to improve the availability of the HAART, CD4+ count and viral load facility worldwide in order to monitor and improve the outcome of patients with neuro-AIDS.

\section{REFERENCES}

1. Deshpande AK, Patnaik MM. Nonopportunistic neurologic manifestations of the human immunodeficiency Virus: An Indian study. J Int AIDS Soc $2005 ; 7: 2$. 
2. Teja VD, Talasila SR, Vemu L. Neurologic manifestations of HIV infection: An Indian hospital-based study. AIDS Read 2005;15:139-43, C3.

3. Fauci AS, Lane HC. Human immunodeficiency virus disease: AIDS and related disorders. In: Kasper DL, Fauci AS, Longo DL, Braunwald E, Hauser SL, Jameson JL, editors. Harrison's Principles of Internal Medicine. $17^{\text {th }}$ ed. USA: The McGraw-Hill Companies Inc.; 2008. p. 1137-203.

4. National AIDS Control Organization, Ministry of Health and Family Welfare, November 2007, Government of India. HIV fact sheets 20032006. Available from: http://www.nacoonline.org/quick_Linkds/HIV_Data/. [Last Accessed 2013 Feb 4]

5. National strategies and algorithm on HIV testing (algorithm III). Guidelines on HIV testing, NACO March 2007. Ministry of health and family welfare. Available from: http://nacoonline.org/upload/Final\%20Publications/ Blood\%20Safety/GUILD.ELINES\%20FOR\%20HIV\%20TESTING.pdf. [Last accessed on 2013 Feb 4]

6. Pant M, Garg P, Seth P. Central Nervous System Infection by HIV-1: Special Emphasis to NeuroAIDS in India. Major Human Viral Infections in India: Current Perspective"Proceedings of the National Academy of Sciences, India. Section B. Biol Sci 2012;82:81-94.

7. Kumarasamy N, Vallabhaneni S, Flanigan TP, Mayer KH, Solomon S. Clinical profile of HIV in India. Indian J Med Res 2005;121:377-94.

8. Kumarasamy N, Solomon S, Flanigan TP, Hemalatha R, Thyagarajan SP, Mayer KH. Natural history of human immunodeficiency virus disease in southern India. Clin Inf Dis 2003;36:79-85.

9. Singh R, Kaur M, Arora D. Neurological complications in late-stage hospitalized patients with HIV disease. Ann Indian Acad Neurol 2011;14:172-7.

10. Thorat SB, Khare KK, Pawar GL, Khadikar SV, Dhar HL. Neurologic manifestation in102 patients admitted with immunosuppression due to HIV infection. Bombay Hosp J 2005;47:354.

11. Satishchandra P, Nalini A, Gourie-Devi M, Khanna N, Santosh V, Ravi V, et al. Profile of neurologic disorders associated with HIV/AIDS from Bangalore, south India (1989-96). Indian J Med Res 2000;111:14-23.

12. Gupta S, Shah DM, Shah I. Neurological disorders in HIV-infected children in India. Ann Trop Paediatr 2009;29:177-81.
13. Bolokadze N, Gabunia P, Ezugbaia M, Gatserelia L, Khechiashvili G Neurological complications in patients with HIVIAIDS. Georgian Med News 2008;165:34-8.

14. Wadhwa A, Kaur R, Bhalla P. Profile of central nervous system disease in HIVIAIDS patients with special reference to cryptococcal infections. Neurologist 2008;14:247-51

15. Jowi JO, Mativo PM, Musoke SS. Clinical and laboratory characteristics of hospitalised patients with neurological manifestations of HIVIAIDS at the Nairobi hospital. East Afr Med J 2007;84:67-76.

16. Verma A. Epidemiology and clinical features of HIV-1 associated neuropathies. J Peripher Nerv Syst 2001;6:8-13.

17. Lanjewar DN, Jain PP, Shetty CR. Profile of central nervous system pathology in patients with AIDS: An autopsy study from India. AIDS 1998;12:309-13.

18. Braicks $O$, Anneken $K$, Reichelt $D$, Schäbitz WR, Dziewas R, Evers $S$, et al. Treatment of neuro-AIDS on a neurological intensive care unit: Epidemiology and predictors of outcome. Nervenarzt 2011;82:1290-5.

19. Hahn K, Husstedt IW; Arendt $G$ für die Deutsche Neuro-AIDSArbeitsgemeinschaft (DNAA). HIV-associated neuropathies. Nervenarzt 2010;81:409-17.

20. Yunis NA, Stone VE. Cardiac manifestations of HIVIAIDS: A review of disease spectrum and clinical management. J Acquir Immune Defic Syndr Hum Retrovirol 1998;18:145-54

21. Sani MU. Myocardial disease in human immunodeficiency virus (HIV) infection: A review. Wien Klin Wochenschr 2008;120:77-87.

Limitations of the study: This was a single-center retrospective study of only neurological manifestations of HIV/AIDS.

How to cite this article: Patil VC, Patil HV. Neurological manifestations of HIV-AIDS at a tertiary care center in western Maharashtra. Int J Med Public Health 2014;4:210-7.

Source of Support: Nil, Conflict of Interest: The authors have no conflicts of interest to declare. 